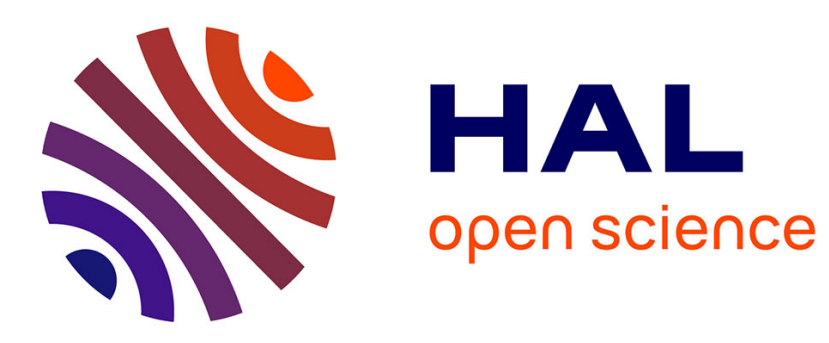

\title{
No-Slip Motion of a Spherical Magnet on the Top of a Conductive Plate
}

\author{
Gérard Vinsard, Esteban Saatdjian, Stéphane Dufour
}

\section{To cite this version:}

Gérard Vinsard, Esteban Saatdjian, Stéphane Dufour. No-Slip Motion of a Spherical Magnet on the Top of a Conductive Plate. IEEE Transactions on Magnetics, 2016, 52 (3), pp.1 - 4. 10.1109/TMAG.2015.2475595 . hal-01498982

\section{HAL Id: hal-01498982 \\ https://hal.univ-lorraine.fr/hal-01498982}

Submitted on 30 Mar 2017

HAL is a multi-disciplinary open access archive for the deposit and dissemination of scientific research documents, whether they are published or not. The documents may come from teaching and research institutions in France or abroad, or from public or private research centers.
L'archive ouverte pluridisciplinaire HAL, est destinée au dépôt et à la diffusion de documents scientifiques de niveau recherche, publiés ou non, émanant des établissements d'enseignement et de recherche français ou étrangers, des laboratoires publics ou privés. 


\title{
No-slip motion of a spherical magnet on top of a conductive plate
}

\author{
Stéphane Dufour, Gérard Vinsard, and Esteban Saatdjian \\ Université de Lorraine, LEMTA, UMR 7563 \\ Vandœuvre-lès-Nancy, TSA60604, F-54518, France
}

\begin{abstract}
The trajectory of a spherical magnet which rolls without slipping on a conductive plate is modelled. A time-stepping $\vec{T}-\Omega$ method is used to find the electromagnetic force and torque on the magnet. Since the degrees of freedom involve both the position and the direction of magnetization of the spherical magnet, the motion here cannot be reduced to the calculation of a single friction force coefficient. The trajectory of the spherical magnet is computed and compared to our experimental data.
\end{abstract}

Index Terms-Conducting materials, eddy currents, finite element methods, magnets.

\section{INTRODUCTION}

$\mathbf{T}$ HE coupling between the partial differential equations (PDEs) that describe the electromagnetic field and the ordinary differential equations (ODEs) that give the motion of a moving conducting object can be a particularly difficult problem especially when the control parameters of the ODEs use variables that issue from the field problems.

Most coupled mechanical-electromagnetic problems concern devices which have only one mechanical degree of freedom [1]-[2]-[3], notable exceptions are magnetohydrodynamical flows. Most cases describe either the field-circuit coupling [4] or the mechanical coupling [5].

If a moving spherical magnet is on top of a conductive plate, eddy currents are induced and tend to slow down the spherical magnet until it stops. This is a transient problem. The position and the magnetization direction are the five mechanical degrees of freedom. The control parameters of the ODEs are the Lorentz force and the torque, which are calculated by solving the transient EM field problem. Similarly, the electromagnetic field depends on the position of the moving magnet. The aim here is to find the dynamics of the magnet, and to validate the accuracy of results by experiments.

Although this is, at first glance, an academic problem, its solution strategy will be used in future work on granular flows. These flows may jam due to Coulomb's friction forces. If spherical magnets, of the same size as the grains, are introduced and submitted to forces like the ones studied here, the flow may be unblocked.

\section{Governing EQUATIONS}

The spherical magnet (radius $R$ ) is assumed to roll without slipping on a horizontal plane. The position $\vec{X}=X(t) \vec{k}_{x}+$ $Y(t) \vec{k}_{y}+R \vec{k}_{z}$ of its center of gravity is obviously time-

Manuscript received June 24, 2015. Corresponding author: G. Vinsard (email: gerard.vinsard@univ-lorraine.fr). We benefited from the savoir faire of Raoul Lenoir in preparing this paper. Financial support from Région Lorraine is also acknowledged. DOI : http://dx.doi.org/10.1108/03321641211267038 0018-9464 (c) 2015 IEEE. Personal use is permitted, but republication / redistribution requires IEEE permission. See http://www.ieee.org/publications_standards/publications/rights/index.html for more information

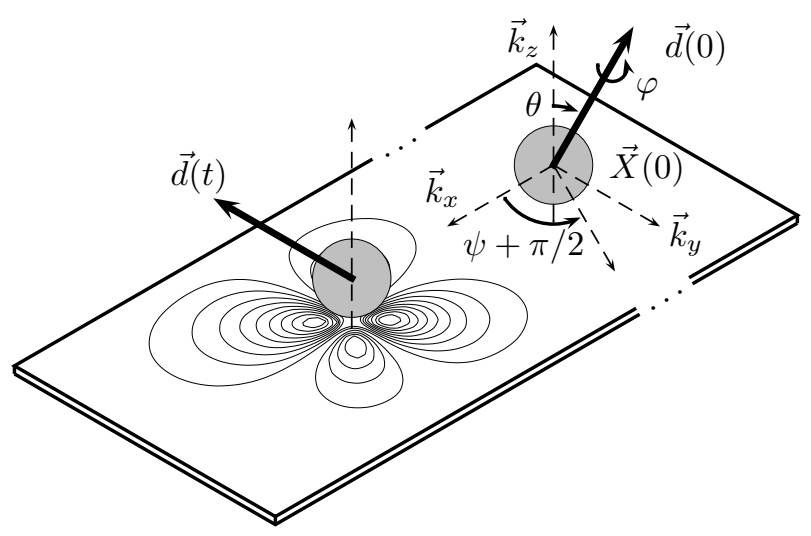

Fig. 1. Sketch of the magnet on top of a conducting plate, the eddy-current lines are also shown on the figure.

dependent, as is the (planar) velocity $\dot{\vec{X}}=\dot{X} \vec{k}_{x}+\dot{Y} \vec{k}_{y}$. The magnetization is directed along

$$
\vec{d}(t)=-\sin \theta \sin \psi \vec{k}_{x}+\sin \theta \cos \psi \vec{k}_{y}+\cos \theta \vec{k}_{z}
$$

where $\psi, \theta$ are the precession and nutation Euler angles (Fig. 1). The intrinsic rotation $\varphi$ around the axis of direction $\vec{d}$ has to be considered to describe the motion of the magnet but has no influence on the magnetic field.

Due to the no-slip condition, the instantaneous rotation vector $\vec{\omega}$ is a function of the planar velocity components with only one extra kinematically free component $\omega_{z}$ :

$$
\vec{\omega}(t)=-\dot{Y}(t) / R \vec{k}_{x}+\dot{X}(t) / R \vec{k}_{y}+\omega_{z}(t) \vec{k}_{z}
$$


Then the motion of the magnet is determined by

$$
\frac{d}{d t}\left(\begin{array}{c}
X \\
Y \\
\dot{X} \\
\dot{Y} \\
\omega_{z} \\
\theta \\
\psi \\
\varphi
\end{array}\right)=\left(\begin{array}{c}
\dot{X} \\
\frac{5}{7 m}\left(f_{x}+\frac{\Gamma_{y}}{R}\right) \\
\frac{5}{7 m}\left(f_{y}-\frac{\Gamma_{x}}{R}\right) \\
\frac{5}{2 m R^{2}} \Gamma_{z} \\
\frac{1}{R}(\dot{X} \sin \psi-\dot{Y} \cos \psi) \\
\omega_{z}+\frac{1}{R \tan \theta}(\dot{X} \cos \psi+\dot{Y} \sin \psi) \\
-\frac{1}{R \sin \theta}(\dot{X} \cos \psi+\dot{Y} \sin \psi)
\end{array}\right)
$$

where $m$ is the mass of the magnet (the apparent mass of a rolling sphere on a plane is $7 \mathrm{~m} / 5$ and its moment of inertia $\left.2 m R^{2} / 5\right)$; and $\vec{f}=f_{x} \vec{k}_{x}+f_{y} \vec{k}_{y}, \vec{\Gamma}=\Gamma_{x} \vec{k}_{x}+\Gamma_{y} \vec{k}_{y}+\Gamma_{z} \vec{k}_{z}$ are the applied force and the torque respectively.

If the viscous and rolling friction are neglected, the force and torque are only due to eddy currents in the copper plate induced by the motion of the magnet.

These eddy-currents are determined by using a timedependent $\vec{T}-\Omega$ formulation. The magnetic field due to the magnet is given by the dipolar expression (outside the magnet):

$$
\vec{h}_{s}=\vec{\nabla}_{\vec{x}}\left(\Omega_{s}\right) ; \Omega_{s}(\vec{x})=-\frac{\mathcal{M} \vec{d} \cdot \overrightarrow{x X}}{4 \pi|\overrightarrow{x X}|^{3}} ; \overrightarrow{x X}=\vec{x}-\vec{X}
$$

where $\mathcal{M}\left(A \mathrm{~m}^{2}\right)$ is the magnetic moment of the magnet. The time derivative of $\vec{h}_{s}$ is $\dot{\vec{h}}_{s}=\vec{\nabla}_{\vec{x}}\left(\dot{\Omega}_{s}\right)$ where

$$
\dot{\Omega}_{s}=\frac{\mathcal{M}}{4 \pi|\overrightarrow{x X}|^{3}} \vec{d} \cdot\left(\dot{\vec{X}}-3 \frac{\dot{\vec{X}} \cdot \overrightarrow{x X}}{|\overrightarrow{x X}|^{2}} \overrightarrow{x X}-\vec{\omega} \times \overrightarrow{x X}\right)
$$

Notice that this term, which is the time-derivative of a kind of magnetomotive force, is a function of all the variables of the dynamical system (3) except $\varphi$.

In the $\vec{T}-\Omega$ formulation, the system of PDEs to be solved both in the conductive plate $D$ and outside the plate $E_{3}-D$ is ( $D$ the conducting domain with a conductivity $\sigma$ and a permeability $\mu_{0}, \partial D$ its boundary and $\vec{n}$ the outward normal field):

$$
\begin{array}{r}
\vec{\nabla} \times\left(\frac{1}{\sigma} \vec{\nabla} \times \vec{T}\right)+\mu_{0} \partial_{t}(\vec{T}+\vec{\nabla} \Omega)+\mu_{0} \dot{\vec{h}}_{s}=\overrightarrow{0} \text { in } D \\
\vec{\nabla} \cdot \vec{T}=\overrightarrow{0} \text { in } D \\
\vec{T} \times \vec{n}=\overrightarrow{0} \begin{array}{l}
\text { on } \partial D ; \quad \vec{T}=\overrightarrow{0} \text { in } E_{3}-D \\
\vec{\nabla} \cdot\left[\mu_{0}(\vec{\nabla} \Omega+\vec{T})\right]=0 \text { in } E_{3}
\end{array}
\end{array}
$$

(6) the time $(n+1) \tau$, the components of the dynamical system (3) (7) system implicitly, but it requires an iterative process to handle

The eddy current density and magnetic field are $\vec{j}=\vec{\nabla} \times \vec{T}$ and $\vec{h}=\vec{T}+\vec{\nabla} \Omega$ and the total Lorentz force and torque exerted on the plate are respectively $\left(d \vec{x}^{3}=d x d y d z\right)$

$$
\begin{aligned}
-\vec{f} & =\int_{D}(\vec{\nabla} \times \vec{T}) \times \mu_{0} \vec{h}_{s} d \vec{x}^{3} \\
-\vec{\Gamma} & =\int_{D} \overrightarrow{x X} \times\left((\vec{\nabla} \times \vec{T}) \times \mu_{0} \vec{h}_{s}\right) d \vec{x}^{3}
\end{aligned}
$$
have to be computed implicitly. It is possible to solve such a

Due to Newton's third law, they are exactly equal and opposite to the force and torque $\vec{f}$ and $\vec{\Gamma}$ exerted on the magnet (labelled $\left.D_{s}\right)$, whose expressions are

$$
\begin{aligned}
& \vec{f}=\int_{\partial D_{s}}-\mu_{0} \mathcal{M}(\vec{d} \cdot \vec{\nabla} \Omega) \vec{n} d \vec{x}^{2} \\
& \vec{\Gamma}=\int_{D_{s}} \mu_{0} \mathcal{M}(\vec{d} \times \vec{\nabla} \Omega) d \vec{x}^{3}
\end{aligned}
$$

The system (6-9) has to be solved in time. A time step $\tau$ is given; $\vec{T}, \Omega$ at discrete times $n \tau$ are denoted $\vec{T}^{n}, \Omega^{n}$ and an estimation $\dot{\vec{h}}_{s}^{* n}$ (described below) of $\dot{\vec{h}}_{s}$ at this time $n \tau$ is used. The full time-implicit weak form version of the $\vec{T}-\Omega$ system (6-7-8) is: $\forall \vec{T}^{\prime}$ such that $\vec{T}^{\prime} \times \vec{n}=\overrightarrow{0}$ on $\partial D$, $\vec{\nabla} \cdot \vec{T}^{\prime}=0$ on $D$

$$
\begin{gathered}
\int_{D}\left(\frac{\vec{\nabla} \times \vec{T}^{n+1}}{\sigma} \cdot \vec{\nabla} \times \vec{T}^{\prime}+\frac{\mu_{0}}{\tau}\left[\vec{T}^{n+1}+\vec{\nabla} \Omega^{n+1}\right] \cdot \vec{T}^{\prime}\right) d \vec{x}^{3} \\
=\int_{D} \frac{\mu_{0}}{\tau}\left[\vec{T}^{n}+\vec{\nabla} \Omega^{n}\right] \cdot \vec{T}^{\prime} d \vec{x}^{3}-\int_{D} \mu_{0} \dot{\vec{h}}_{s}^{* n+1} \cdot \vec{T}^{\prime} d \vec{x}^{3}
\end{gathered}
$$

Practically the constraint $\vec{\nabla} \cdot \vec{T}=0$ in $D$ is taken into account by an extra penalty term. Since the only contribution of $\vec{\nabla} \cdot \vec{T}$ in $E_{3}$ is the product between the jump of $\vec{T} \cdot \vec{n}$ on $\partial D$ and the surface Dirac distribution, the Biot and Savart form of (9) reduces to:

$$
\Omega^{n+1}(\vec{x})=\frac{\mu_{0}}{4 \pi} \int_{\partial D} \frac{\vec{T}^{n+1}(\vec{y}) \cdot \vec{n}}{|\vec{x}-\vec{y}|} d \vec{y}^{2}
$$

Even if $\Omega$ can be computed in $E_{3}$, the computation is only required in the domain $D$ to find $\vec{T}$ with (12). It allows us to use a fixed mesh (and then to disregard the motion of the magnet in the mesh) as well as a fixed frame of reference.

For a given $\dot{\vec{h}}_{s}$ at the time $(n+1) \tau$, the computation of $\left(\vec{T}^{n+1}, \Omega^{n+1}\right)$ can be performed from a given set of $\left(\vec{T}^{n}\right.$, $\left.\Omega^{n}\right)$ by (12-13) with the finite element method. To avoid handling a non-sparse matrix, (12-13) is computed iteratively: $\vec{T}^{n+1}$ is computed with (12) for a given $\Omega^{n+1}$, and $\Omega^{n+1}$ with (13) for a given $\vec{T}^{n+1}$. Practically, if the time step is not too large, convergence is obtained with a limited number of iterations, which depends on the used convergence criterium. The computation of (13) is done once and the corresponding matrix is stored. The singularities contained by (13) are handled with closed-form expressions.

If $\dot{\vec{h}}_{s}^{* n+1}$ really corresponds to $\dot{\vec{h}}_{s}^{n+1}$, the value of $\dot{\vec{h}}_{s}$ at the non-linearities. An estimation of $\left(\vec{X}^{n+1}, \vec{d}^{n+1}, \vec{\omega}^{n+1}\right)$ is first required to compute $\dot{\vec{h}}_{s}^{n+1}$, then $\vec{T}^{n+1}$ is obtained. The forces and torques are deduced and an implicit scheme of (3) is used to find a new estimate of $\left(\vec{X}^{n+1}, \vec{d}^{n+1}, \vec{\omega}^{n+1}\right)$. Such a method of solution would be time-consuming due to the numerous degrees of freedom (including the $\vec{T}-\Omega$ ones), and the fact that the problem is not time-periodic, even if diagonally implicit Runge-Kutta (DIRK) methods were to be used [6]-[7].

To overcome this drawback, a kind of semi-implicit method is used: the system of ODEs (3) is solved explicitly, whereas 
(12-13) is solved partially implicitly. $\left(\vec{X}^{n+1}, \vec{d}^{n+1}, \vec{\omega}^{n+1}\right)$ are found with a time step of (3) from $\left(\vec{X}^{n}, \vec{d}^{n}, \vec{\omega}^{n}\right)$, with an explicit high order Runge-Kutta method (Dormand-Prince 5), where the components of force and torque computed with (10) are frozen at time $n \tau$, i.e. computed with $\left(\vec{T}^{n}, \vec{h}_{s}^{n}\right)$. Then, as $\left(\vec{X}^{n+1}, \vec{d}^{n+1}, \vec{\omega}^{n+1}\right)$ are considered as given, $\dot{\vec{h}}_{s}^{* n+1}$ can be deduced as $\dot{\vec{h}}_{s}\left(\vec{X}^{n+1}, \vec{d}^{n+1}, \vec{\omega}^{n+1}\right)$. (12-13) are then solved implicitly with the source term $\dot{\vec{h}}_{s}^{* n+1}$, which allows to find $\left(\vec{T}^{n+1}, \Omega^{n+1}\right)$.

Let us introduce the magnetic co-energy $\bar{W}$, Joule losses $p_{j}$, given supplied electrical power $p_{s}$ of induced currents

$$
\begin{aligned}
\bar{W} & =\int_{E_{3}} \mu_{0}(\vec{T}+\vec{\nabla} \Omega)^{2} d \vec{x}^{3} ; p_{j}=\int_{D} \frac{(\vec{\nabla} \times \vec{T})^{2}}{\sigma} d \vec{x}^{3} \\
p_{s} & =-\int_{D} \mu_{0} \dot{\vec{h}}_{s} \cdot \vec{T} d \vec{x}^{3}
\end{aligned}
$$

and kinetic energy $E_{k}$, supplied mechanical power $p_{m}$ to the magnet

$$
E_{k}=\frac{m}{2}\left(\dot{\vec{X}}^{2}+\frac{2}{5} R^{2} \vec{w}^{2}\right) ; p_{m}=\vec{f} \cdot \overrightarrow{\dot{X}}+\vec{\Gamma} \cdot \vec{\omega}
$$

The weak forms of (6-9) and the Hamiltonian form of the dynamical system (3) allow us to write two half-power balance equations:

$$
\frac{d \bar{W}}{d t}+p_{j}=p_{s} ; \frac{d E_{k}}{d t}=p_{m}
$$

The coupling which results from the dependence of $\dot{\vec{h}}_{s}$ on mechanical variables $(5)$ and of $(\vec{f}, \vec{\Gamma})$ on electrical variables (10) corresponds to:

$$
p_{s}+p_{m}=0 \Longrightarrow \frac{d}{d t}\left(\bar{W}+E_{k}\right)+p_{j}=0
$$

At the discrete level, the two-half power balances (16) are globally satisfied. But, due to the approximation on $\dot{\vec{h}}_{s}$, this is less so for the total balance equation (17), the accuracy of this equality has to be monitored.

\section{RESULTS}

A spherical Nd-Fe-B magnet $\left(R=6.35 \mathrm{~mm}, \mathcal{M}=1 \mathrm{Am}^{2}\right)$ is thrown on a copper plate (thickness $5 \mathrm{~mm}, \sigma=50 \mathrm{MS} / \mathrm{m}$ ), by using a helical compression spring. It helps to ensure a reliable initial speed $\vec{X}(t=0)=\dot{x}_{0} \vec{k}_{x}\left(\dot{x}_{0}=3.7 \mathrm{~m} / \mathrm{s}\right)$. Even if it is a low effet negligible during it motion, due to the earth's magnetic field the equilibrium direction of magnetization of the motionless spherical magnet is $\theta_{0}=25^{\circ}$ in Nancy (France), which fixes one initial condition. The other one can be chosen as $\psi_{0}=0$, for which the shot direction is perpendicular to the north-south axis. The trajectory of the magnet has been filmed with a high-speed camera (500 frames $/ \mathrm{s}, 6 \mathrm{px} / \mathrm{mm})$. Figure 2 shows snapshots of the magnet from $t=0$ to $t=100 \mathrm{~ms}$, every $20 \mathrm{~ms}$. The terminal position, $t=160 \mathrm{~ms}$, is represented by the cross.

The motion of the magnet has been computed with the help of (3-12-13) and compared with the experiment, to determine the validity of the model (Fig. 3). The model (plain line) is in a good agreement with the experimental data (dashed line):

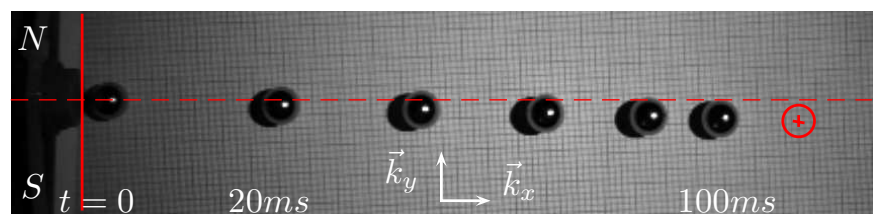

Fig. 2. Snapshots of the magnet at a set of discrete times $(\Delta t=20 \mathrm{~ms})$.

the difference between the computed abscissa $X$ travelled, and the measured one is less than $4 \%$, for a total travelled distance of $24 \mathrm{~cm}$.

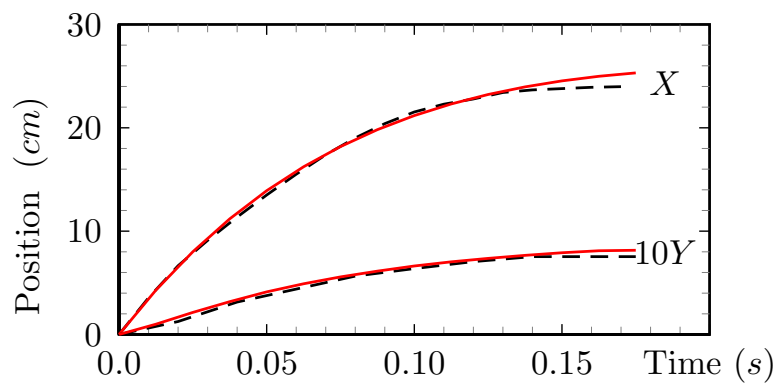

Fig. 3. Position $(X, Y)$ of the magnet vs time (dashed line: experimental, plain line: computed).

As can be seen on Fig. 3, in addition to the main motion along $\vec{k}_{x}$, there is also a motion of much smaller amplitude along $\vec{k}_{y}$ (about $0.8 \mathrm{~cm}$ ). We have checked that this does not occur with a non-magnetic sphere. This lateral motion is due to the fact that $\theta_{0} \neq 90^{\circ}$. If this were not the case, the starting direction would be along $\vec{k}_{y}$, and $Y$ would remain at zero.

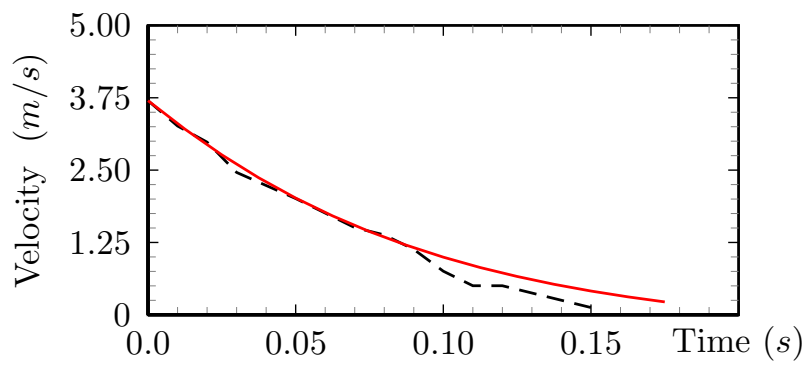

Fig. 4. Velocity $\dot{X}$ vs time (dashed line: experimental, plain line: computed).

The experimental velocity $\dot{X}$ has been estimated with the change in position between snapshots (Fig. 4 dashed line). We have checked that the motion of the spherical magnet cannot be obtained with a single viscous-friction force along the $x$-direction.

The computed velocity $\dot{X}$ (Fig. 4 plain line) is of the same order of magnitude. At the end of the motion, there is a slightly higher difference between computation and experiment, two reasons are invoked. The first is the earth's magnetic field $\vec{b}_{e}$, whose torque $\vec{\Gamma}_{e}=\int_{D_{s}} \mathcal{M}\left(\vec{d} \times \vec{b}_{e}\right) d \vec{x}^{3}$ has to be taken into account. The intensity of the magnetic field due to the eddy currents is compared with the intensity of the earth's magnetic field $(0.05 \mathrm{mT})$ (Fig. 5). Although this effect is not completely negligible, it is too small to explain the difference. The magnet's rotation due to the earth's magnetic field occurs 


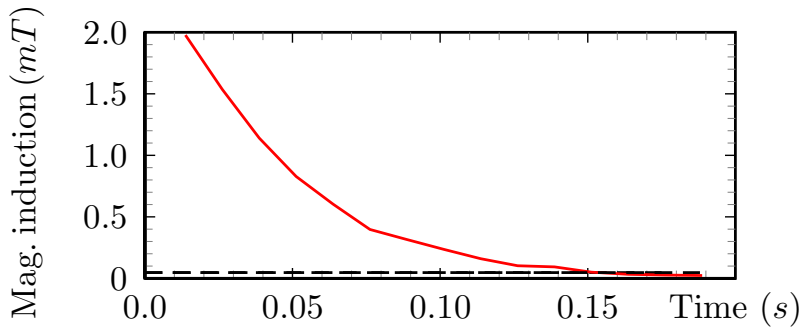

Fig. 5. Magnetic induction due to the eddy currents in the center of the magnet $\mu_{0}|\vec{\nabla} \Omega|$ vs time (plain line) and earth magnetic induction (dashed line).

in experiments when $t=150 \mathrm{~ms}$ : both fields are of the same order of magnitude. The second reason is the frictional contact: the rolling friction (or adhesion phenomenon) plays a role when the velocity is low (An analogous phenomenon has been observed with a non-magnetic sphere ; we have not investiged further due to the complexity of these tribology aspects).

The chosen time step is $\tau=1.2 \mathrm{~ms}$. In the worst case, when the velocity is a maximum $(3.7 \mathrm{~m} / \mathrm{s})$, it corresponds to 8 steps per rotation of the sphere. This is sufficient to ensure accuracy with a 5th-order Runge-Kutta method and does not lead to a too long time of calculation ( 2 hours with a $2.8 \mathrm{GHz}$ processor). To verify this statement we have computed the difference

$$
E_{k}(t)-E_{k}(0)-\int_{0}^{t} p_{m}(\tau) d \tau
$$

and have obtained an error less than $0.1 \%$ at the end of the motion $\left(E_{k}(0)\right.$ is the initial energy stored in the spring). If $-p_{s}$ is substituted to $p_{m}$ in (18), a bigger error is obtained, but it is less than $0.8 \%$ (Fig. 6): it validates the assumption of using $\dot{\vec{h}}_{s}^{* n+1}$ instead of the true values $\dot{\vec{h}}_{s}^{n+1}$ in the coupling (forces and torques are given at each time step).

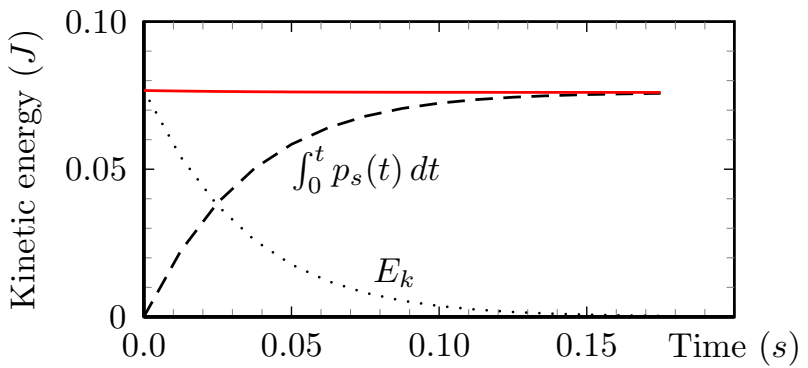

Fig. 6. Kinetic energy $E_{k}$ (dotted line), work of the electrical force (dashed line) and sum of both terms (plain line) versus time.

Finally the starting direction of magnetization is a parameter which affects the trajectory. In the case considered here, the shot direction was chosen perpendicular to the north-south axis $\left(\psi_{0}=0^{\circ}\right)$. This introduces a symmetry default which generates a slight deviation along $\vec{k}_{y}$ but $\vec{d}$ becomes quickly aligned to $\vec{k}_{y}$ and then remains parallel to the plate.

If now a symmetric configuration is chosen, where the shot direction is aligned to the north-south axis (and then the initial direction of magnetization is in the $\left(\vec{k}_{x}, \vec{k}_{z}\right)$ plane), a more complex kinematics is obtained (Fig. 7). When the initial velocity is high $(3.7 \mathrm{~m} / \mathrm{s})$ there is a complex rotating motion during the third of the time that leads $\vec{d}$ to be aligned with $\vec{k}_{y}$. But when this initial velocity is low $(1 \mathrm{~m} / \mathrm{s}), \vec{d}$ remains in the $\left(\vec{k}_{x}, \vec{k}_{z}\right)$ plane: the sphere rolls without deviating.

The stopping distance ( $X$ when the magnetic field due to the induced current becomes equal to the terrestrial magnetic field) is approximately $24 \mathrm{~cm}$ for the case $\left(\psi_{0}=0^{\circ}, 3.7 \mathrm{~m} / \mathrm{s}\right)$; this distance is $20 \%$ smaller in the case $\left(\psi_{0}=-90^{\circ}, 3.7 \mathrm{~m} / \mathrm{s}\right)$. The same comparison made with an initial velocity $1 \mathrm{~m} / \mathrm{s}$ gives a ratio of $50 \%$. The same ratios have been obtained by experiment.

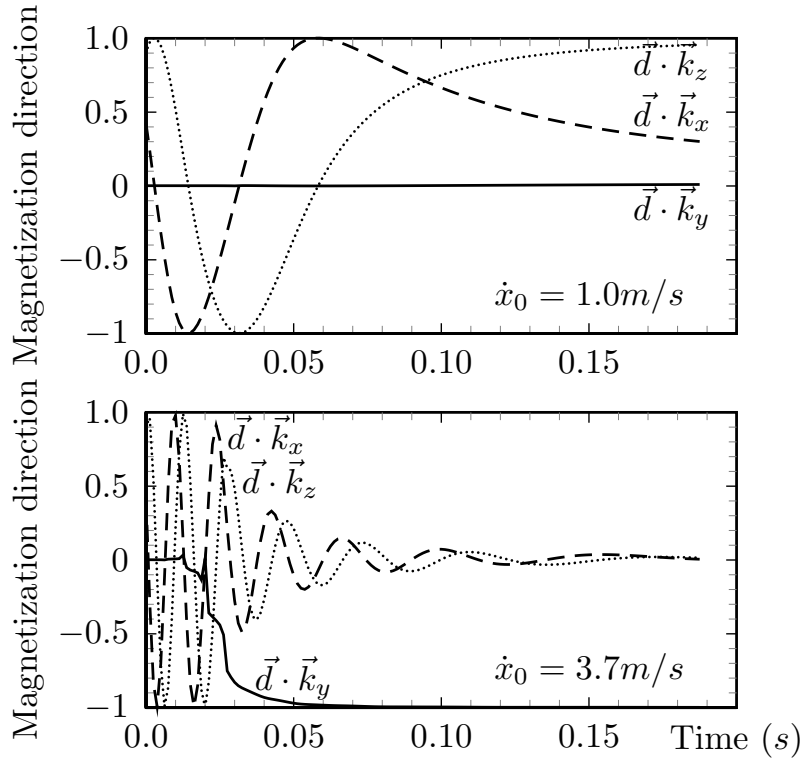

Fig. 7. Components of the magnetization direction vs time $\left(\vec{d} \cdot \vec{k}_{y}\right.$ in plain line, $\vec{d} \cdot \vec{k}_{x}$ dashed line, $\vec{d} \cdot \vec{k}_{z}$ dotted line). Initial conditions: $\psi_{0}=-90^{\circ}, \theta_{0}=$ $25^{\circ} ; \dot{x}_{0}=3.7 \mathrm{~m} / \mathrm{s}$ (bottom) and $\dot{x}_{0}=1.0 \mathrm{~m} / \mathrm{s}$ (top).

\section{CONCLUSION}

The major aim here was to solve a problem where an electromagnetic field is coupled to the motion of a solid particle. The difficulties lie essentially in the choice of the approximations on the coupling variables: force and torque on one side, position and speed on the other side. We decided to freeze these variables during a time-step for each problem, even if the accuracy of the numerical schemes differ. Comparison with experiment shows that this approach is adequate. In order to reduce the computation time, an embedded RungeKutta method with an energy criterion (difference between kinetic energy and accumulated electric power) will be tested.

\section{REFERENCES}

[1] C. Weidermann, I. Sokolov and A. Thess, "Lorentz Force and Joule Heat Induced in an Electrically Conducting Plate Moving With TimeDependent Velocity Under the Influence of a Homogeneous Magnetic Field" IEEE Trans. Mag., vol. 50, no. 8, Aug. 2014.

[2] M. Zec, R.P. Uhlig, M. Ziolkowski and H. Brauer, "Fast Technique for Lorentz Force Calculations in Non-destructive Testing Applications" IEEE Trans. Mag., vol. 50, no. 2, Feb. 2014.

[3] N. Paudel and J.Z. Bird, "General 2-D Steady-State Force and Power Equations for a Traveling Time-Varying Magnetic Source Above a Conductive Plate" IEEE Trans. Mag., vol. 48, no. 1, Jan. 2012. 
[4] E. Melgoza, C.A. Cruz, V. Venegas, R. Escarela-Perez and J.L. Guardado, "Strong Coupling of Electromagnetic Transients and Finite Element Magnetic Field Solvers", IEEE Trans. Mag., vol. 47, no. 11, pp. 45744581, Nov. 2011.

[5] A. Carpentier, N. Galopin, O. Chadebec, and G. Meunier, "Modeling of Magneto-Mechanical Coupling Using Magnetic Volume Integral and Mechanical Finite-Element Methods", IEEE Trans. Mag., vol. 50, no. 2, Feb. 2014.

[6] A. Nicolet and F. Delincé, "Implicit Runge-Kutta Methods for Transient Magnetic Field Computation", IEEE Trans. Mag., vol. 32, no. 3, May 1996.

[7] J. Pries and H. Hofmann, "Steady-State Algorithms for Nonlinear TimePeriodic Magnetic Diffusion Problems Using Diagonally Implicit RungeKutta Methods", IEEE Trans. Mag., vol. 51, no. 4, Apr. 2015. 